\title{
How should sparse marine in situ measurements be compared to a continuous model: an example
}

\author{
L. de Mora, M. Butenschön, and J. I. Allen \\ Plymouth Marine Laboratory, Prospect Place, The Hoe, Plymouth, PL1 3DH, UK \\ Correspondence to: L. de Mora (ledm@pml.ac.uk) \\ Received: 18 July 2012 - Published in Geosci. Model Dev. Discuss.: 20 August 2012 \\ Revised: 22 March 2013 - Accepted: 4 April 2013 - Published: 25 April 2013
}

\begin{abstract}
This work demonstrates an example of the importance of an adequate method to sub-sample model results when comparing with in situ measurements. A test of model skill was performed by employing a point-to-point method to compare a multi-decadal hindcast against a sparse, unevenly distributed historic in situ dataset. The point-to-point method masked out all hindcast cells that did not have a corresponding in situ measurement in order to match each in situ measurement against its most similar cell from the model. The application of the point-to-point method showed that the model was successful at reproducing the inter-annual variability of the in situ datasets. Furthermore, this success was not immediately apparent when the measurements were aggregated to regional averages. Time series, data density and target diagrams were employed to illustrate the impact of switching from the regional average method to the point-topoint method. The comparison based on regional averages gave significantly different and sometimes contradicting results that could lead to erroneous conclusions on the model performance. Furthermore, the point-to-point technique is a more correct method to exploit sparse uneven in situ data while compensating for the variability of its sampling. We therefore recommend that researchers take into account for the limitations of the in situ datasets and process the model to resemble the data as much as possible.
\end{abstract}

\section{Introduction}

Numerical models are now used extensively in earth, climate and ocean sciences. Furthermore, numerical models are frequently used to inform policy decisions. Both policy decision and fundamental science require models to have demonstrable quality. However, the assessment of how well a model captures reality is an ongoing challenge of marine ecosystem model development.

As discussed in a meta-analysis (Arhonditsis and Brett, 2004), many models have been validated with qualitative methods exclusively. Qualitative methods are usually straightforward to interpret, allowing for a simple, fast, subjective judgement of whether the model appears to be representative of the measurements. Unfortunately, a model may seem to recreate emergent properties and well-known largescale features of the ecosystem, yet struggle to reproduce the historic data of a hindcast, for instance Doney et al. (2009). For these reasons, it is crucial to validate models using a variety of objective statistical tests.

In marine ecosystem modelling, quantitative descriptions of models are often based on pattern statistics, and other univariate indices, for instance in Stow et al. (2009). Pattern statistics form the axes of the popular Taylor (Taylor, 2001) and target diagrams (Jolliff et al., 2009). These univariate indices generally require equal binning of (i.e. the same number of) both model and measurement data, but the methodology used to achieve equal binning can introduce sampling bias.

Typically, the equal binning condition is met by interpolating the data to cover the model domain. Interpolations fill under-sampled regions with information from well-sampled regions - an ideal solution for cloud coverage in satellite data (for instance Edwards et al., 2012). However, interpolating sparse, uneven, widely distributed three-dimensional in situ measurements can amplify the effects of sampling bias (Robeson, 1994). This is especially true for measurements with high spatial and temporal variability. Furthermore, due to the complex nature of the water columns' vertical 
structure, it is extremely difficult to interpolate sparse marine in situ measurements at depth into a three-dimensional grid. Typically, 3-D interpolation assumes that all axes have equal weight, but in the ocean, the vertical scale varies in distances of metres and the horizontal scales in kilometres. It is not straightforward to choose whether a pixel should be more influenced by a deeper nearby point or by a distant point of similar depth.

Alternatively, the equal binning condition can be achieved by taking the mean of both the model and in situ data over a sufficiently large region, as in Lewis et al. (2006). When the data are three-dimensional marine in situ measurements, choosing an appropriate mean can be a challenge as the mean of an arbitrary set of marine in situ measurements in three dimensions is unlikely to be a good indicator of the typical state of the system. Further sampling bias is introduced when the mean of measurements of a very small subset of the ocean is compared against the mean of a very large volume in the model. These problems are compounded when ad hoc sampling is further biased toward coastal sites that are both accessible and convenient.

In this paper, a point-to-point method is outlined to validate a marine ecosystem model hindcast in using historic in situ measurements in the politically significant North Sea region. The point-to-point method does not introduce new uncertainties via interpolation and attempts to reduce the impact of sampling bias introduced via historic ad hoc sampling. While it may seem obvious to process the model exactly as the in situ data were produced, it seems to have rarely been done in marine biogeochemical modelling, or published with a lack of transparency. Furthermore, to the best of the authors' knowledge, this is the first direct comparison of the matched against unmatched methodologies.

The model used in this study is the European Regional Seas Ecosystem Model (ERSEM) coupled with the Proudman Oceanographic Laboratory Coastal-Ocean Modelling System (POLCOMS) as described in Sects. 2 and 3. The in situ data are the Conductivity, Temperature and Depth (CTD) sampler data and low-resolution bottle data in the North Sea from the International Council of the Sea (ICES) database, described in Sect. 4. A full description of the methodology of the point-to-point matching and the linear regression is in Sect. 5. The agreement of the in situ and model data, and a comparison of matched and unmatched methods are shown in Sect. 6.

\section{Circulation model}

The Proudman Oceanographic Laboratory Coastal-Ocean Modelling System (POLCOMS) hydrodynamic model (Holt et al., 2001) is a baroclinic three-dimensional model that includes both the deep ocean and the continental shelf.

This study used POLCOMS-ERSEM in the Atlantic Margin Model (AMM) domain, which covers the area between $40^{\circ} \mathrm{N}$ to $65^{\circ} \mathrm{N}$ and $20^{\circ} \mathrm{W}$ to $13^{\circ} \mathrm{E}$. The domain has a resolution of $1 / 9^{\circ}$ by $1 / 6^{\circ}$, which equates to $12 \mathrm{~km}$ with a baroclinic timestep of $15 \mathrm{~min}$. In terms of depth, the s-coordinates system is used, consisting of 40 wet depth layers of varying thickness.

The atmospheric boundary conditions were taken from the ERA 40 reanalysis (Uppala et al., 2005) between 1960 and September 2001; subsequent conditions were from the ECMWF operational analysis. As described in Holt et al. (2012), the atmospheric air temperature, wind, pressure and relative humidity, daily precipitation and short-wave radiation were used in surface forcing in six-hourly intervals. The open ocean boundary conditions were taken from the global model, ORCA025. The freshwater fluxes in the AMM consist of 250 rivers from the Global River Discharge Database (Vörösmarty et al., 2000) and from the Centre for Ecology and Hydrology.

The model was run for a $45 \mathrm{yr}$ hindcast between 1960 and 2004, with each state variable recorded as the daily and monthly mean. However, the model spins up until 1970, so the period between 1960-1970 is not considered in this analysis. A full description of POLCOMS-ERSEM in the AMM domain is available in Holt et al. (2012).

\section{Biogeochemistry model}

POLCOMS was coupled to the European Regional Seas Ecosystem Model, ERSEM. ERSEM is a lower-trophic level biogeochemical cycling model that uses the functional-group approach (Blackford et al., 2004). The carbon, nitrogen, oxygen, phosphorus, and silicon cycles are explicitly resolved, and the food web is composed of four phytoplankton, three zooplankton and one bacterial functional type.

The nutrients and oxygen forcing were taken from World Ocean Atlas Data (Garcia and Levitus, 2010a,b). The river nutrient content is based on measured data, as in Holt et al. (2012) and Young and Holt (2007). The Baltic exchange at the Belts is treated as an inflow source using a mean annual cycle of depth-averaged transport, salinity and nutrients.

\section{In situ data}

The in situ data used in this study were taken from the the International Council for the Exploration of the Sea (ICES) EcoSystemData Online Warehouse (ICES, 2009). Five datasets were used: temperature, salinity, nitrates, phosphates, and chlorophyll $a$. This study aimed to have good spatial and temporal coverage and consistent data quality. For these reasons, only bottle and low-resolution CTD data were used.

The region under investigation, the North Sea as defined by the ICES subdivision, IV (FAO, 2008), and the boundary of the AMM domain are shown in Fig. 1. The North Sea region was chosen because of the quantity and regularity of 


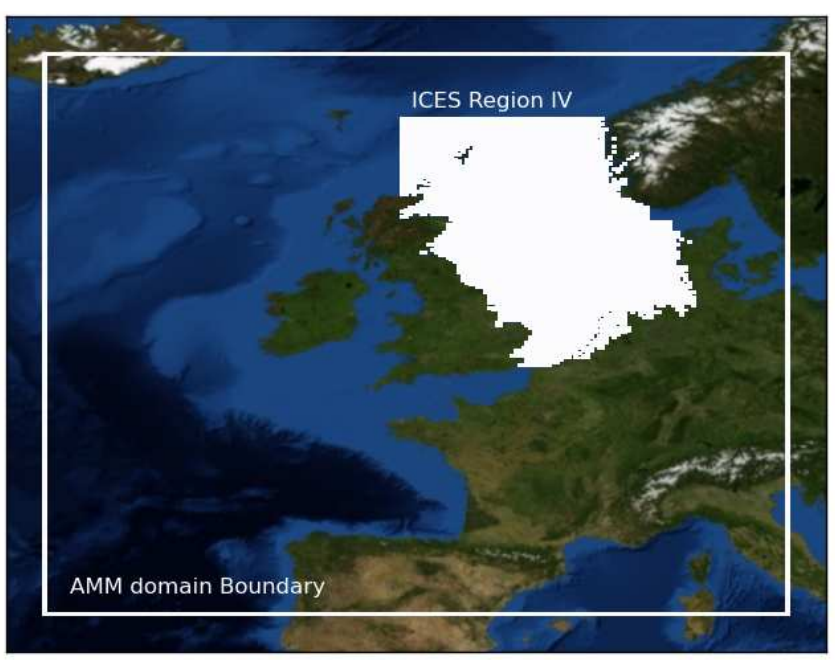

Fig. 1. The Atlantic Margin Model (AMM) boundary and the North Sea as defined by ICES region IV.

data there, but also because the North Sea is sufficiently distant from the edge of the AMM domain that it should not suffer from open ocean boundary condition distortions. Furthermore, data-based validation of the POLCOMS-ERSEM model in the North Sea region is an important policy-driven task. Additionally, there was a computational upper limit on the size of the matching database; larger datasets covering large spatial regions required non-trivial computational resources.

The North Sea ICES region is defined as the sea between $62^{\circ} \mathrm{N}$ and $51^{\circ} \mathrm{N}$, and $4^{\circ} \mathrm{W}$. The eastern boundary of the North Sea domain passes north from Agger Tange, Jutland, Denmark, to $57^{\circ} \mathrm{N}$, west to $8^{\circ} \mathrm{E}$, then north to $57^{\circ} 30^{\prime} \mathrm{N}$, then west to $7^{\circ} \mathrm{E}$, then north to the coast of Norway.

The in situ data were provided in a comma-separatedvariable format, and contained a few data quality anomalies, such as repeated data, which were addressed during processing. The repeated measurements accounted for typically $10-20 \%$ of the database. Repeated data were identified by searching for measurements with identical measurement time, longitude, latitude, depth and value. In some cases, data were recorded at depths much greater than the model bathymetry at the same point, so measurements with a depth greater than $5 \mathrm{~m}$ below the model bathymetry at the same point were ignored. The chlorophyll dataset contained a large proportion of measurements with a value of exactly 0.1 , even at depths below $1 \mathrm{~km}$. As no chlorophyll is expected at large depths, this was interpreted to be the detection limit of the database and all chlorophyll measurements below $100 \mathrm{~m}$ were removed from the study.

\section{Methods}

This section describes the methods that were used to test the compatibility of the model and the in situ data. The first subsection describes how the point-to-point matching was applied, the second the difference between time selection and time granularity, and the third the linear regression fit.

\subsection{Point-to-point matching}

The model and in situ datasets are intrinsically dissimilar. The model dataset is formed from a grid of continuous, evenly distributed, time-averaged cells of approximately $12 \mathrm{~km}$ by $12 \mathrm{~km}$. The in situ dataset is a series of sporadic, unevenly distributed, instantaneous measurements from a CTD or the mean of the contents of a sampling bottle. Furthermore, the in situ measurements tended to occur at times and places which were readily accessible, convenient or well funded. These places and times do not match the uniform grid used by the model. The role of point-to-point matching was to reduce the impact of sampling bias when comparing these two distinct kinds of data.

The first step of the point-to-point matching process was performing a snap-to-grid: the in situ data were collected into the same grid cells as the model. Here, the full depth, daily mean, four-dimensional AMM domain grid was used. This grid had 40 depth layers, 198 longitude bins and 224 latitude bins per day. In the rare cases when multiple in situ measurements fell into the same three-dimensional grid cell on the same day, the mean of those measurements was used. Otherwise, the same model pixel could appear multiple times in the matched dataset.

Finally, all model pixels that did not have a corresponding in situ measurement were masked out and vice versa. In this way, no unpaired model or in situ data were used in the linear regression.

Techniques similar to this point-to-point matching have been used elsewhere in geoscientific models, but they are rare in marine ecosystem modelling. For instance, in Jöckel et al. (2010), a dataset was created during the model run that matched a specific flight path with the highest model time resolution available. Similarly, in Lewis et al. (2006), Continuous Plankton Recorder tracks were extracted from a POLCOMS-ERSEM run for 1989-1990 in the North Sea. Unfortunately, the typical time required to produce a $45 \mathrm{yr}$ POLCOMS-ERSEM hindcast is on the order of one month and implementing this technique could double the run time requirement. For this reason, run time methods of data recording were beyond the scope of this work.

While it may seem obvious that model and in situ measurements match better when the domains match better, Saux Picart et al. (2012) demonstrated that this is not the case and that the current generation of POLCOMS-ERSEM does not perform equally well on all scales. In fact, Saux-Picart et al. (2012) found that the model performed better on larger 


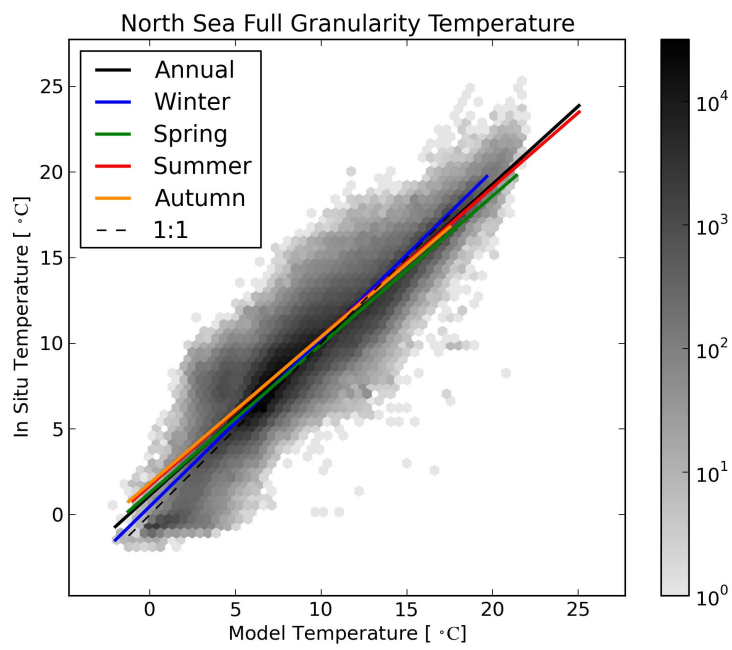

Fig. 2. Two-dimensional binned scatter plot of full granularity matched model data against in situ measurements for the North Sea temperature. The solid coloured lines show the linear regression fits for the full annual and seasonal data, and the dashed line is the line of unity slope that passes through the origin. The shading of the binned data density plot is scaled such that darker hue indicates logarithmically higher data density.

spatial scales than smaller ones for chlorophyll, with relatively poor skill when matching against satellite data on small spatial scales.

\subsection{Time selection and granularity}

It is important to highlight the difference between time selection and time granularity. Once the matching was performed, both model and in situ datasets were studied under two time granularities: the annual mean and the daily mean. They were also studied under five different time selections: annual, winter, spring, summer and autumn. All ten permutations of the two granularities and the five time selections were studied.

The time granularity defines how that data are aggregated, if at all. The daily or "full" granularity refers to a dataset containing every matched pair of points in the North Sea, and the "annual" granularity is a series of annual means of that dataset. Typically, the full dataset contains many thousand matched pairs of in situ and model data, whereas the annual mean datasets contain only 35 points: one for every year between 1970 and 2005 . The annual time granularity allows the study of inter-annual variability in nature, in the model and in the sampling bias. The "full" granularity allows the comparison of each in situ measurement to its model counterpart, and is used for identifying the limitations of the model.

The time selection specifies which part of the year is studied. As the AMM is a Northern Hemisphere domain, the winter time selection masked out all measurements that did not occur in January, February or March. Similarly, the spring contains the data from April, May and June; the summer

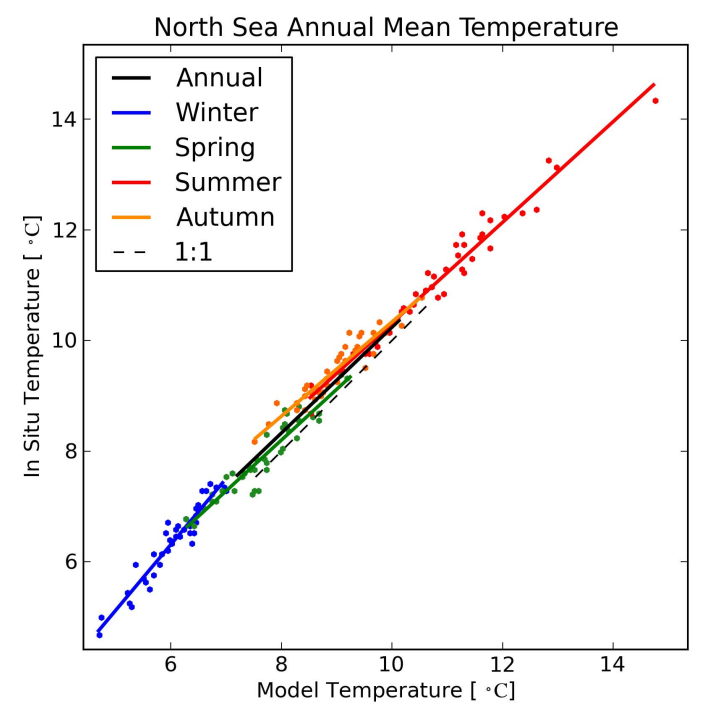

Fig. 3. Two-dimensional scatter plot of annual mean matched model data against in situ measurements for the North Sea temperature. The solid coloured lines show the linear regression fits for the annual and seasonal annual means data, and the dashed line is the line of unity slope that passes through the origin. The matched seasonal means are shown as colour-coded scatter points.

contains the data from July, August and September; the autumn contains the data from October, November and December. The annual time selection effectively means that no time selection was made and that data from all times of the year are used; it is sometimes referred to as "no time selection".

As a shorthand, each combination of time selection and granularity can be described using the time granularity followed by the selection: for instance, full winter or annual spring. The specific case of the annual granularity and annual selection is called the annual mean.

\subsection{Linear regression}

The relationship between model data and in situ measurements was plotted with the model on the $\mathrm{x}$-axis and the in situ data on the y-axis, then fitted to a straight line using a least-squares linear regression. This technique minimises the sum of the square of the residuals; the residuals are the difference between a matched data pair and the closest point on the linear regression line. The five output parameters of the regression were the following: the y-axis intersect $\left(\hat{\beta}_{0}\right)$, the slope of the fit $\left(\hat{\beta}_{1}\right)$, the standard error $(\epsilon)$, the correlation coefficient $(R)$ and the two-tailed probability $(P)$. The best possible outcome of linear regression, corresponding to a perfect model, would be a line of slope unity through the origin with no standard error. The two-tailed probability, $P$ or $p$ value, is the probability that these data are not derived by chance. The $p$ values are not a measure of goodness of fit; they are a measure of the confidence that the linear regression 
Table 1. Linear regression output parameters for temperature.

\begin{tabular}{lccccc}
\hline Name & Full & Full Winter & Full Spring & Full Summer & Full Autumn \\
\hline$\hat{\beta}_{1}$ & 0.9083 & 0.979 & 0.866 & 0.8704 & 0.8549 \\
$\hat{\beta}_{0}$ & 1.131 & 0.4908 & 1.28 & 1.701 & 1.83 \\
$R$ & 0.9363 & 0.8637 & 0.894 & 0.9417 & 0.8598 \\
$P$ & $<10^{-4}$ & $<10^{-4}$ & $<10^{-4}$ & $<10^{-4}$ & $<10^{-4}$ \\
$\epsilon$ & 0.0003 & 0.0011 & 0.0008 & 0.0006 & 0.001 \\
$N$ & 1191530 & 271599 & 334676 & 308946 & 276309 \\
\hline \multirow{2}{*}{ Name } & Annual & Annual Winter & Annual Spring & Annual Summer & Annual Autumn \\
\hline$\hat{\beta}_{1}$ & 0.9565 & 1.192 & 0.9183 & 0.9101 & 0.8524 \\
$\hat{\beta}_{0}$ & 0.6754 & -0.8403 & 0.8538 & 1.215 & 1.813 \\
$R$ & 0.9572 & 0.9602 & 0.942 & 0.9814 & 0.9445 \\
$P$ & $<10^{-4}$ & $<10^{-4}$ & $<10^{-4}$ & $<10^{-4}$ & $<10^{-4}$ \\
$\epsilon$ & 0.0469 & 0.0563 & 0.0531 & 0.0289 & 0.0481 \\
\hline
\end{tabular}

Table 2. Linear regression output parameters for salinity.

\begin{tabular}{lccccc}
\hline Name & Full & Full Winter & Full Spring & Full Summer & Full Autumn \\
\hline$\hat{\beta}_{1}$ & 1.245 & 1.292 & 1.281 & 1.199 & 1.183 \\
$\hat{\beta}_{0}$ & -8.305 & -9.977 & -9.641 & -6.854 & -6.262 \\
$R$ & 0.7681 & 0.7466 & 0.7843 & 0.8067 & 0.7169 \\
$P$ & $<10^{-4}$ & $<10^{-4}$ & $<10^{-4}$ & $<10^{-4}$ & $<10^{-4}$ \\
$\epsilon$ & 0.001 & 0.0022 & 0.0018 & 0.0016 & 0.0022 \\
$N$ & 1176225 & 264732 & 333987 & 303397 & 274109 \\
\hline Name & Annual & Annual Winter & Annual Spring & Annual Summer & Annual Autumn \\
\hline$\hat{\beta}_{1}$ & 1.268 & 1.176 & 1.31 & 1.096 & 1.152 \\
$\hat{\beta}_{0}$ & -9.221 & -6.023 & -10.63 & -3.298 & -5.178 \\
$R$ & 0.7558 & 0.7742 & 0.9212 & 0.8115 & 0.8103 \\
$P$ & $<10^{-4}$ & $<10^{-4}$ & $<10^{-4}$ & $<10^{-4}$ & $<10^{-4}$ \\
$\epsilon$ & 0.1783 & 0.1559 & 0.0897 & 0.128 & 0.1351 \\
\hline
\end{tabular}

output parameters are correct, under the assumption that all data points have an equal influence on the fit. As such, given a matched dataset with some relationship between the in situ and the model, the $p$ value tends to decrease with large sample size, even when the relationship is weak or non-linear.

\section{Results}

The results of the linear regression fits are shown in Tables 15. These tables hold the results of the linear regression for the North Sea temperature $(T)$, salinity (Sal.), nitrates $\left(\mathrm{NO}_{3}\right)$, phosphates $\left(\mathrm{PO}_{4}\right)$ and chlorophyll (Chl.) datasets. Each table contains both the full and the annual time granularity for each of the five time selections, as described in Sect. 5.2. The rows of these tables contain the five output parameters of the regression: the slope of the fit $\left(\hat{\beta}_{1}\right)$, the $y$-axis intersect $\left(\hat{\beta}_{0}\right)$, the correlation coefficient $(R)$, the two-tailed probability $(P)$, the standard error $(\epsilon)$, and the number of data in the sample $(N)$. The number of data in the sample, $N$, is the total number of data pairs that contributed to the linear regression. However, in the case of the annual means, the number of entries that were regressed is 35 or less, equivalent to one entry for each year (1970-2005), and $N$ is not shown.

Figures 2-11 are two-dimensional scatter plots for the temperature, salinity, nitrates, phosphates and chlorophyll matched datasets. These figures were prepared for each matched dataset with the model data plotted as the $\mathrm{x}$ coordinate and the in situ data as the y-coordinate. The best fit linear regression line for each time selection is shown as a solid coloured line, and the parameters of these fits are held in Tables 1-5. These figures also all have a dashed black line that represents the line of slope unity that passes through the origin. The dashed black line divides the figure into two regions; the model underestimates the in situ measurements in 
Table 3. Linear regression output parameters for nitrates.

\begin{tabular}{lccccc}
\hline Name & Full & Full Winter & Full Spring & Full Summer & Full Autumn \\
\hline$\hat{\beta}_{1}$ & 1.05 & 1.267 & 0.9105 & 0.6153 & 0.8885 \\
$\hat{\beta}_{0}$ & -4.248 & -8.121 & -1.355 & -1.016 & -3.409 \\
$R$ & 0.5928 & 0.6584 & 0.4567 & 0.3728 & 0.5548 \\
$P$ & $<10^{-4}$ & $<10^{-4}$ & $<10^{-4}$ & $<10^{-4}$ & $<10^{-4}$ \\
$\epsilon$ & 0.0042 & 0.0082 & 0.0102 & 0.0101 & 0.0074 \\
$N$ & 116933 & 31575 & 30243 & 22892 & 32223 \\
\hline Name & Annual & Annual Winter & Annual Spring & Annual Summer & Annual Autumn \\
\hline$\hat{\beta}_{1}$ & 0.6872 & 1.336 & 0.6463 & 0.6168 & 0.7163 \\
$\hat{\beta}_{0}$ & 0.2063 & -10.05 & 0.9477 & -0.8275 & -0.843 \\
$R$ & 0.8132 & 0.8191 & 0.7484 & 0.5798 & 0.7207 \\
$P$ & $<10^{-4}$ & $<10^{-4}$ & $<10^{-4}$ & 0.0001 & $<10^{-4}$ \\
$\epsilon$ & 0.0798 & 0.1518 & 0.0929 & 0.1406 & 0.1118 \\
\hline
\end{tabular}

Table 4. Linear regression output parameters for phosphates.

\begin{tabular}{lccccc}
\hline Name & Full & Full Winter & Full Spring & Full Summer & Full Autumn \\
\hline$\hat{\beta}_{1}$ & 0.6823 & 0.7882 & 0.4098 & 0.4736 & 0.9168 \\
$\hat{\beta}_{0}$ & 0.1802 & 0.0993 & 0.2373 & 0.3293 & 0.0199 \\
$R$ & 0.4153 & 0.4926 & 0.2583 & 0.2211 & 0.4462 \\
$P$ & $<10^{-4}$ & $<10^{-4}$ & $<10^{-4}$ & $<10^{-4}$ & $<10^{-4}$ \\
$\epsilon$ & 0.0043 & 0.0077 & 0.0087 & 0.0134 & 0.01 \\
$N$ & 121860 & 32957 & 31231 & 24153 & 33519 \\
\hline Name & Annual & Annual Winter & Annual Spring & Annual Summer & Annual Autumn \\
\hline$\hat{\beta}_{1}$ & 0.1814 & 0.938 & 0.2687 & 0.0152 & 0.8202 \\
$\hat{\beta}_{0}$ & 0.4929 & -0.0255 & 0.3015 & 0.532 & 0.1253 \\
$R$ & 0.1283 & 0.5799 & 0.2179 & 0.0082 & 0.5982 \\
$P$ & 0.43 & 0.0001 & 0.1769 & 0.9598 & 0.0001 \\
$\epsilon$ & 0.2274 & 0.2167 & 0.1952 & 0.2991 & 0.1806 \\
\hline
\end{tabular}

the top left region and overestimates the in situ data in the lower right region. In addition to the lines of best fit for the full granularities, Figs. 2, 4, 6, 8 and 10 also show the full granularity matched data as a binned scatter plot. The shading of the binned scatter plots is scaled such that darker hue indicates logarithmically higher data density. However, the linear regression fits were performed using a non-logarithmic scale. Figures 3, 5, 7, 9 and 11 show the linear regressions for each annual granularity time selection and also contain colour-coded scatter plots of the annual means for each of the four seasons.

The linear regression lines in Figs. 2-11 were drawn with a horizontal and vertical range between the smallest and largest value of either the matched model or the in situ data. This method makes comparison between time selections relatively straightforward, but it can sometimes be misleading. For instance, the darker high density regions of Figs. 2, 4, 6, 8 and
10 all coincide with the line of best fit and the line of slope unity through the origin. However, the best fit lines appear to diverge from the matched points in the lower data density regions.

Temperature was especially well reproduced by POLCOMS-ERSEM, as shown in Figs. 2 and 3. Even with more than one million matched pairs of model and in situ data in Fig. 2, a correlation of $R>0.9$ is observed in the full annual linear regression. Furthermore, this correspondence between model and measurement is visible for all time selections. The seasonality of the model and data is especially visible in Fig. 3, where there is little overlap between the four seasonal means, yet all time selections have equivalently good performance. In all combinations of time selection and granularity in Table 1, the correlation between model and in situ temperature was high, while the $p$ value and the standard error were both very low. As 
Table 5. Linear regression output parameters for chlorophyll.

\begin{tabular}{lccccc}
\hline Name & Full & Full Winter & Full Spring & Full Summer & Full Autumn \\
\hline$\hat{\beta}_{1}$ & 0.7479 & 0.8829 & 0.5341 & 0.9941 & 3.391 \\
$\hat{\beta}_{0}$ & 2.052 & 1.165 & 2.952 & 1.98 & 0.7616 \\
$R$ & 0.2379 & 0.2132 & 0.186 & 0.23 & 0.2609 \\
$P$ & $<10^{-4}$ & $<10^{-4}$ & $<10^{-4}$ & $<10^{-4}$ & $<10^{-4}$ \\
$\epsilon$ & 0.0171 & 0.05 & 0.0264 & 0.0418 & 0.2 \\
$N$ & 32019 & 6552 & 11406 & 10121 & 3940 \\
\hline Name & Annual & Annual Winter & Annual Spring & Annual Summer & Annual Autumn \\
\hline$\hat{\beta}_{1}$ & 1.098 & 0.1448 & 1.62 & 3.031 & 4.514 \\
$\hat{\beta}_{0}$ & 1.139 & 1.839 & 0.2675 & -0.0887 & 0.7984 \\
$R$ & 0.2844 & 0.0632 & 0.37 & 0.7083 & 0.3444 \\
$P$ & 0.0793 & 0.7492 & 0.0263 & $<10^{-4}$ & 0.0674 \\
$\epsilon$ & 0.6085 & 0.4483 & 0.6975 & 0.5515 & 2.369 \\
\hline
\end{tabular}

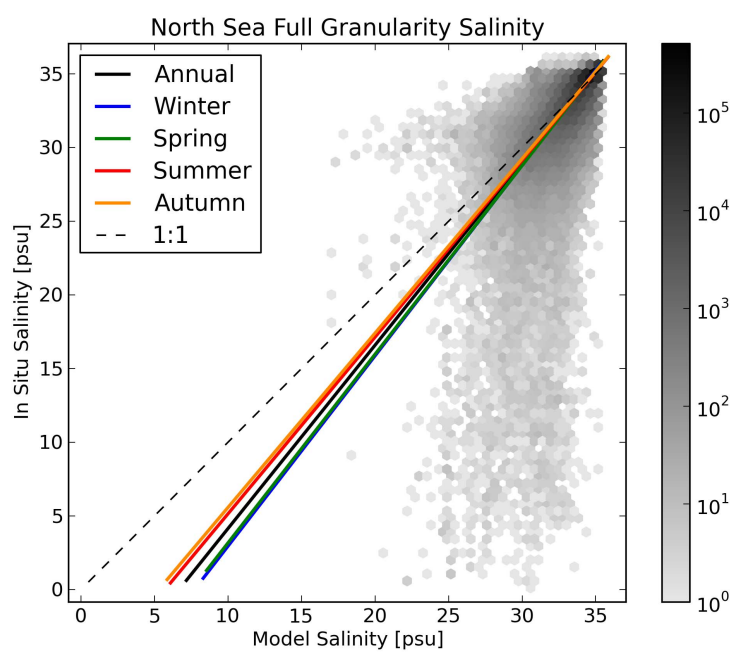

Fig. 4. Two-dimensional binned scatter plot of full granularity matched model data against in situ measurements for the North Sea salinity. The solid coloured lines show the linear regression fits for the full annual and seasonal data, and the dashed line is the line of unity slope that passes through the origin. The shading of the binned data density plot is scaled such that darker hue indicates logarithmically higher data density.

marine models tend to be more successful at simulating physics than biology, temperature is the variable where the best correlation is expected. Furthermore, ocean temperature has much less variability over small temporal and spatial scales than the biological observables.

The salinity plots in Figs. 4 and 5 show good agreement between in situ and model for the regions of high density, and that these regions coincide with the line of slope unity through the origin. These plots are a good example of how the difference between the ranges of the full and the annual

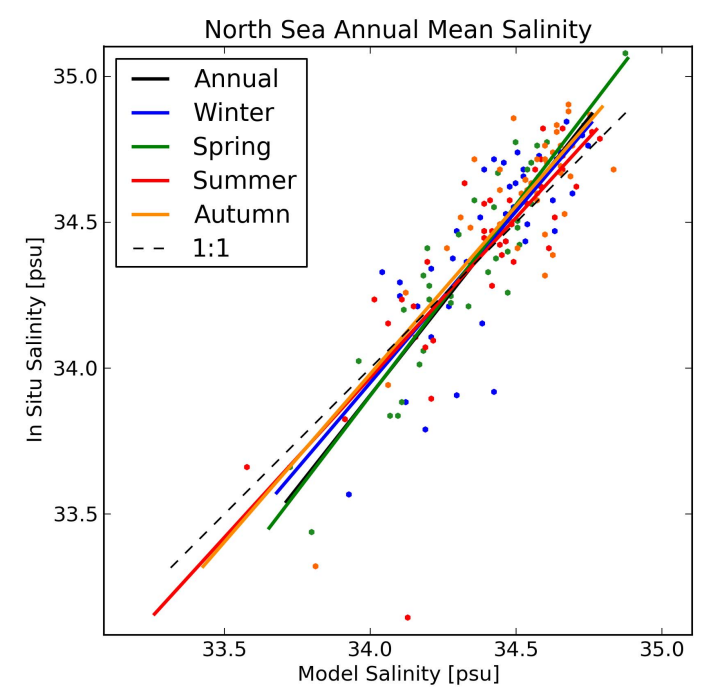

Fig. 5. Two-dimensional scatter plot of annual mean matched model data against in situ measurements for the North Sea salinity. The solid coloured lines show the linear regression fits for the annual and seasonal annual means data, and the dashed line is the line of unity slope that passes through the origin. The matched seasonal means are shown as colour-coded scatter points.

plots impacts the perceived model quality. The full in situ data range between 0 and $36 \mathrm{psu}$, whereas the annual mean data has a much tighter range between 33 and 35 psu. This indicates that the bulk of the in situ data are well matched, even though a cursory glance at Fig. 4 might give the opposite impression.

The two-dimensional data density salinity plot in Fig. 4 also illustrates a limitation of the point-to-point method. The point-to-point method is not ideal in situations where the model pixel size is much greater than the scale of variability 


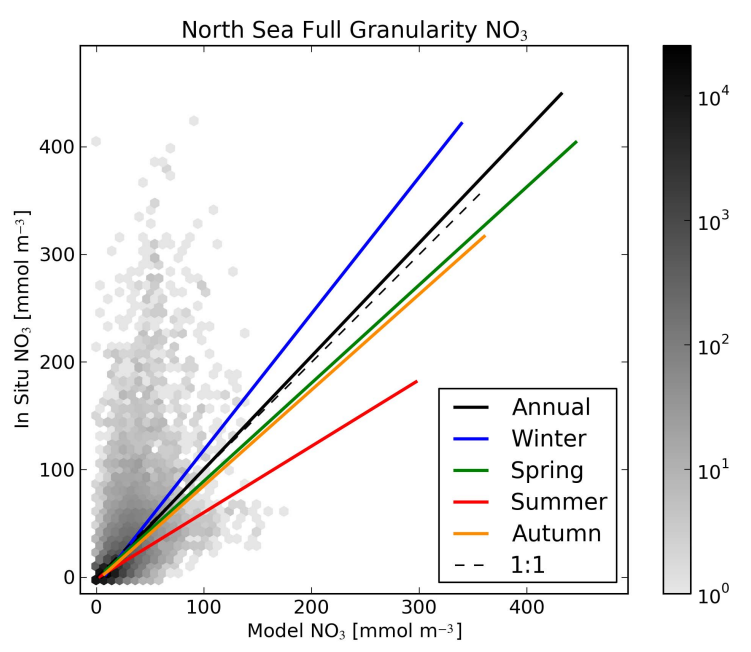

Fig. 6. Two-dimensional binned scatter plot of full granularity matched model data against in situ measurements for the North Sea nitrates. The solid coloured lines show the linear regression fits for the full annual and seasonal data, and the dashed line is the line of unity slope that passes through the origin. The shading of the binned data density plot is scaled such that darker hue indicates logarithmically higher data density.

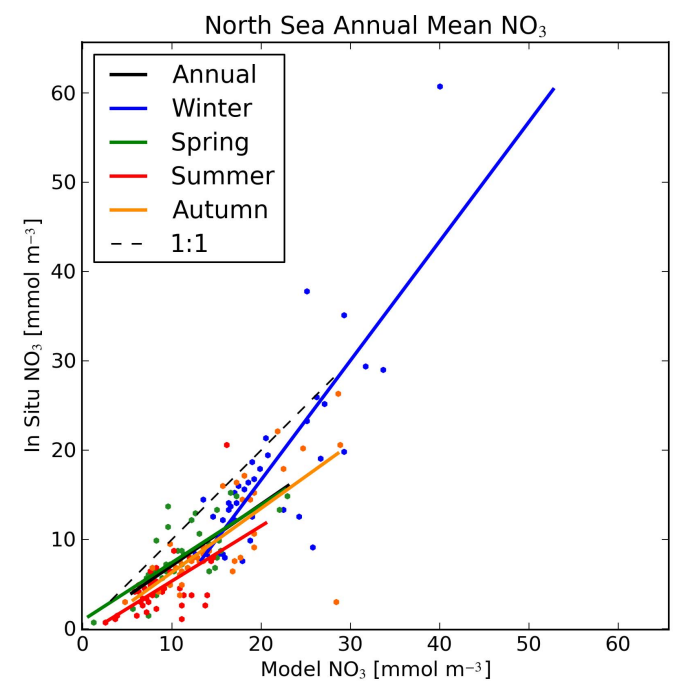

Fig. 7. Two-dimensional scatter plot of annual mean matched model data against in situ measurements for the North Sea nitrates. The solid coloured lines show the linear regression fits for the annual and seasonal annual means data, and the dashed line is the line of unity slope that passes through the origin. The matched seasonal means are shown as colour-coded scatter points.

of the measurements. For instance, at the confluence of a river and the sea, the salinity may range from nearly $0 \mathrm{psu}$ near the river mouth to 35 psu $10 \mathrm{~km}$ away in the sea. However, this model has $12 \mathrm{~km}$ by $12 \mathrm{~km}$ pixel size, and all measurement data between the river mouth and $10 \mathrm{~km}$ offshore

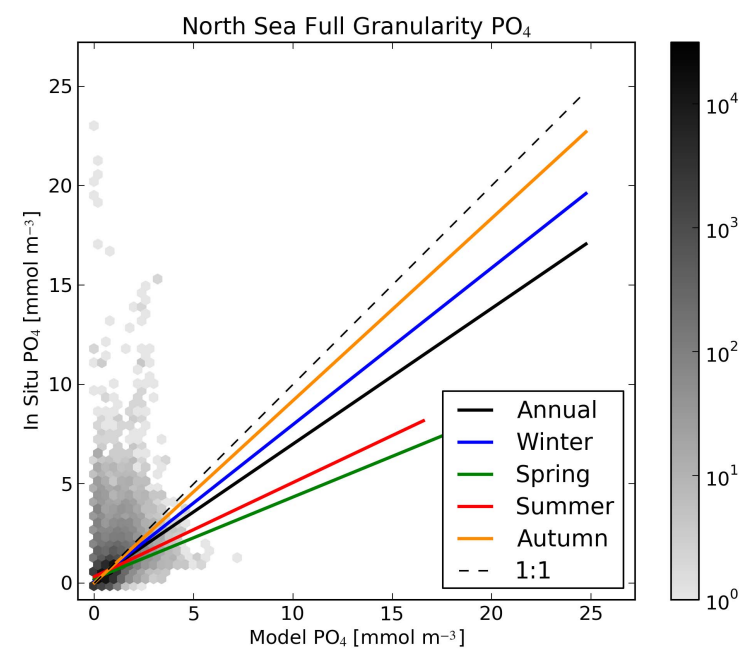

Fig. 8. Two-dimensional binned scatter plot of full granularity matched model data against in situ measurements for the North Sea phosphates. The solid coloured lines show the linear regression fits for the full annual and seasonal data, and the dashed line is the line of unity slope that passes through the origin. The shading of the binned data density plot is scaled such that darker hue indicates logarithmically higher data density.

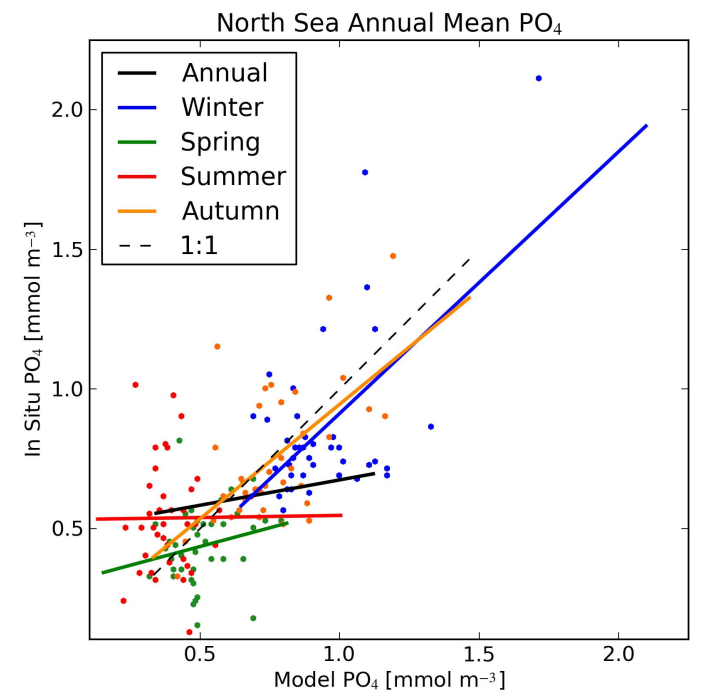

Fig. 9. Two-dimensional scatter plot of annual mean matched model data against in situ measurements for the North Sea phosphates. The solid coloured lines show the linear regression fits for the annual and seasonal annual means data, and the dashed line is the line of unity slope that passes through the origin. The matched seasonal means are shown as colour-coded scatter points.

fall into the same model pixel. This may be one of the reasons why the model appears to overestimate the salinity of many freshwater in situ measurements. As such, point-topoint matching is not ideal for studying datasets and regions with high sub-pixel variability. 


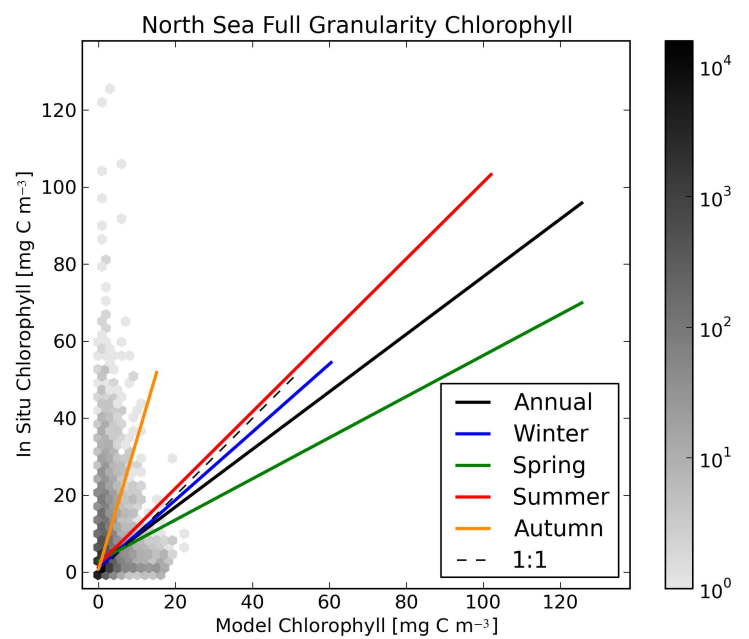

Fig. 10. Two-dimensional binned scatter plot of full granularity matched model data against in situ measurements for the North Sea chlorophyll. The solid coloured lines show the linear regression fits for the full annual and seasonal data, and the dashed line is the line of unity slope that passes through the origin. The shading of the binned data density plot is scaled such that darker hue indicates logarithmically higher data density.

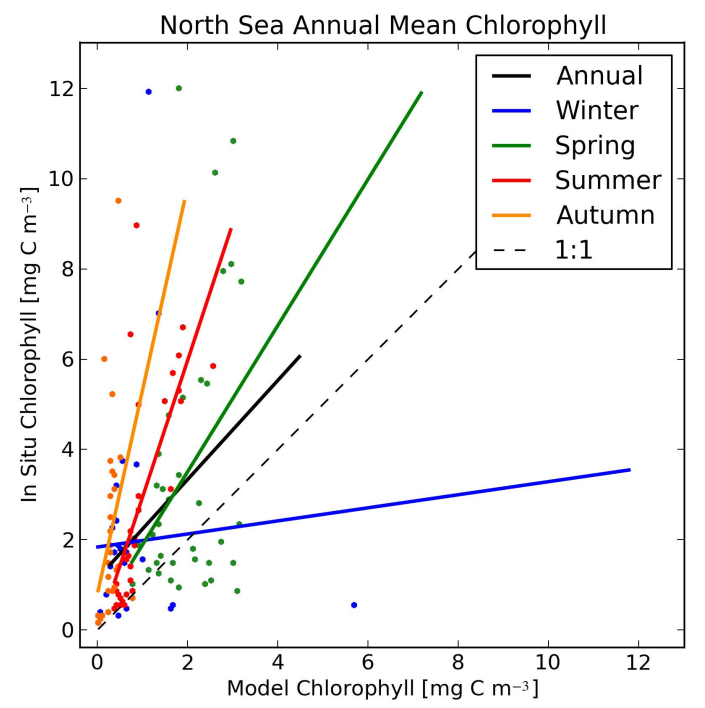

Fig. 11. Two-dimensional scatter plot of annual mean matched model data against in situ measurements for the North Sea chlorophyll. The solid coloured lines show the linear regression fits for the annual and seasonal annual means data, and the dashed line is the line of unity slope that passes through the origin. The matched seasonal means are shown as colour-coded scatter points.

By using the point-to-point matching method, it becomes possible to identify some limitations of the model. For instance, the off-diagonal regions of the salinity, nitrates and phosphates full data density plots in Figs. 4, 6 and 8 contain significantly fewer data than the densely populated

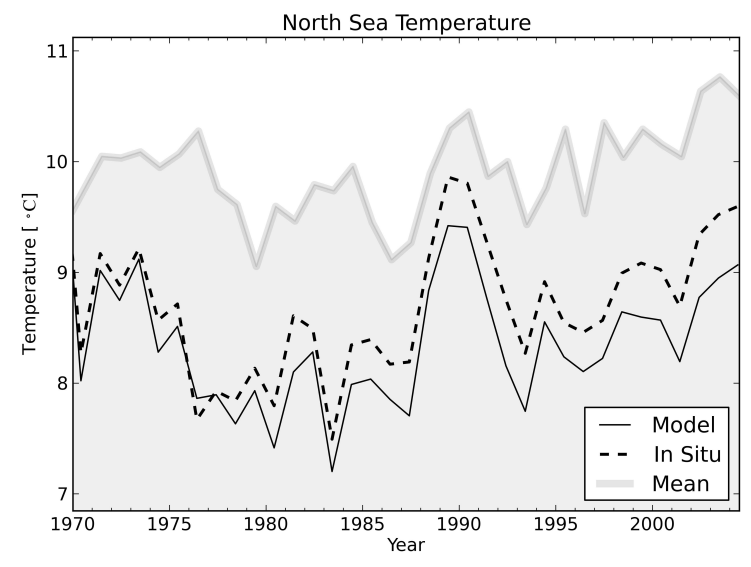

Fig. 12. Annual North Sea temperature time series plot: the black line is the matched model mean annual temperature, the dashed line the annual mean in situ temperature, and and the grey line shows the MLD-averaged mean temperature of the North Sea.

on-diagonal hot spots. However, the presence of any points at all away from the dashed line indicates that the model did not accurately reproduce all the in situ measurements. The model overestimated the salinity of many freshwater in situ measurements and underestimated many of the in situ measurements with high nitrates and phosphates. In both cases, the model predicted a value less extreme than the outlying in situ measurement. Some of these discrepancies can be explained as an effect of the high spatial variability in salinity and nitrates in the well-sampled coastal and riverinfluenced regions against the relatively low spatial resolution of the model. In addition, the in situ data are of instantaneous character, while the model data are a daily average, further enhancing the in situ measurement variability. Conversely, Figs. 4, 6 and 8 all contain a small number of points where the opposite situation occurred: the model salinity was underestimated, and the nitrate and phosphate concentrations were overestimated. These data suggest that there may be some events in the river forcing where the model has a larger influx of fresh water and nutrients than was observed in nature.

The seasonal nitrate and phosphate scatter plots in Figs. 7 and 9 shows that the model captures the seasonality of the nutrient cycle with winter peaks and a spring and summer depletion. However, it is important to bear in mind that these data are not indicative of the mean state of the system. Rather, these figures indicate that the nutrient seasonality is apparent in both model and data despite the data used being an arbitrary subsection of measurements of the North Sea.

Figures 10 and 11 are the full and annual linear regression plots for the North Sea chlorophyll. The full granularity chlorophyll data density plot in Fig. 10 shows that there are some similarities to the case of the nutrients: the model underestimates the extreme in situ measurements, captures the 


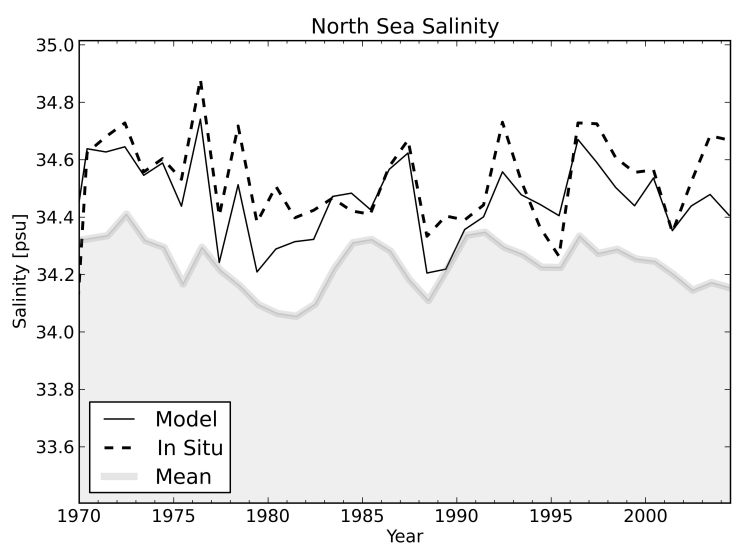

Fig. 13. Annual North Sea salinity time series: the black line is the matched model mean annual salinity, the dashed line the mean annual in situ salinity, and the grey area shows the MLD-averaged mean salinity of the North Sea.

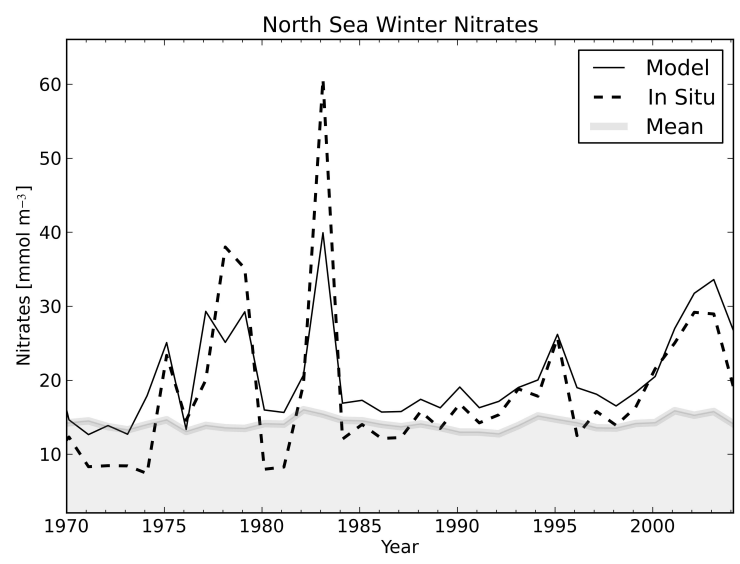

Fig. 14. North Sea mean winter nitrates time series: the black line is the matched model mean winter nitrates, the dashed line the mean winter in situ nitrates, and the grey area shows the MLD-averaged mean nitrate of the North Sea.

the densely populated on-diagonal hot spots, but also has a region where the model overestimates the in situ chlorophyll.

A significant difference between the full and the annual data appears in Figs. 10 and 11. The full plot (Fig. 10) shows that the spring, winter and annual model data tend to overestimate the in situ chlorophyll in the fit, while the summer fit is close to the $1: 1$ line. However, the annual mean chlorophyll plot (Fig. 11) indicates that the annual, autumn, summer and spring chlorophyll are underestimated by the model. Once again, it is important to remember that the in situ dataset, and hence the model data, is an arbitrary subsection of the North Sea.

The ICES chlorophyll database has been amalgamated from a wide selection of sources, using multiple measurement techniques, whose uncertainties vary from more to less validated. Of the five datasets studied here, chlorophyll

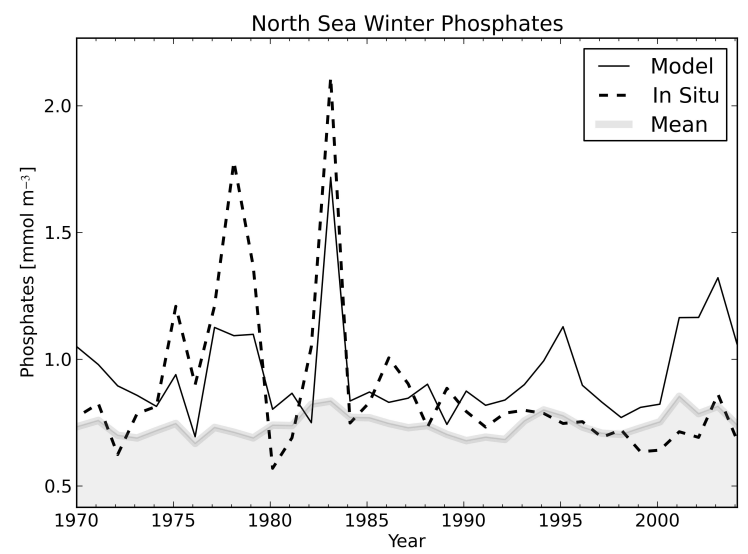

Fig. 15. North Sea mean winter phosphates time series: the black line is the matched model mean winter phosphates, the dashed line the mean winter in situ phosphates, and the grey area shows the MLD-averaged mean phosphates of the North Sea.

typically has the highest measurement uncertainty. Additionally, in order for a model to capture the phytoplankton behaviour, it must first model the physics and nutrient dynamics appropriately. The model errors and uncertainties are compounded with each step away from a tractable physics observable towards the biological end of the model. As such, chlorophyll is the dataset with the highest in situ measurement uncertainty and with the highest model uncertainty. While there is not an excellent agreement between model and the in situ chlorophyll in Figs. 10 and 11, there is a good enough agreement.

Figures 12-17 are the time series plots for annual temperature, annual salinity, annual winter nitrates, annual winter phosphates, annual spring chlorophyll and annual summer chlorophyll. These combinations of variables and time selections were chosen because of their value in informing policy and model validation. These figures each contain three curves: the matched model data (black line), the in situ data (dotted line), and the mixed layer depth-averaged (MLDaveraged) of the North Sea in the model (grey area). The North Sea model MLD-averaged region plots are the model data in the North Sea region before any data were masked by the point-to-point matching method. This is included to estimate whether the matched and in situ variation correspond to overall trends, or sampling biases. The MLD-averaged data are included to illustrate both an example of a previous way to perform this study and as a cross reference in order to test if there is some inter-annual changes in mean state of the North Sea in the model that could be hidden by the patchiness of the in situ dataset. The annual mean time granularity was selected for all these plots and tables such that the matched data could be compared fairly against the MLDaveraged data, which was not possible to produce under the full time granularity. In all cases, the inter-annual variability of the mean of the MLD-averaged is smaller than that of 


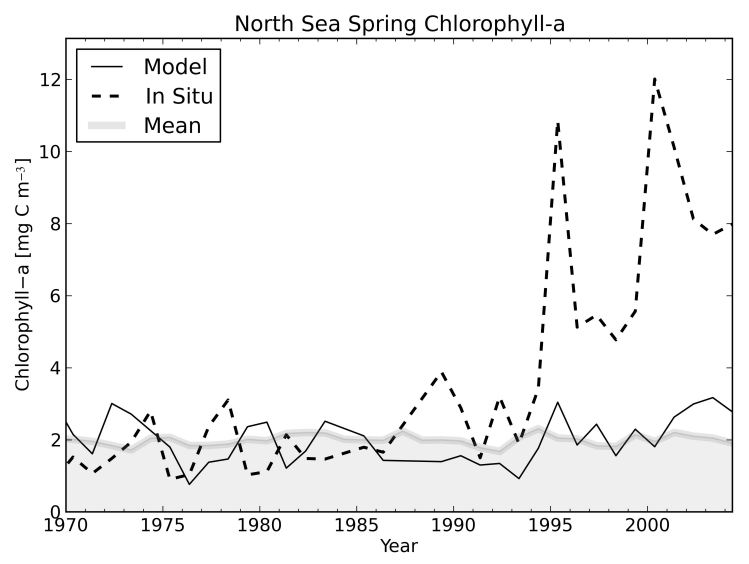

Fig. 16. North Sea mean spring chlorophyll time series: the black line is the matched model mean spring chlorophyll, the dashed line the mean spring in situ chlorophyll, and the grey area shows the MLD-averaged mean chlorophyll of the North Sea.

the matched model data and the in situ measurements, especially in the case of nitrates, phosphates and chlorophyll in Figs. 14-17.

The results of the linear regressions of Figs. 12-17 are shown in Tables 6 and 7. These tables have two columns: the results of the linear regression of the MLD-averaged model data against the in situ, and the linear regression of the matched data against the in situ data. In all cases shown here, the matching resulted in a higher correlation coefficient, decreased $p$ value, and the $y$-intersect closer to zero. In all cases shown except summer chlorophyll, matching results in a slope closer to unity than the MLD-averaged linear regression. The fit for each dataset is discussed in more detail below.

Figure 12 is a time-series plot of the annual mean North Sea temperature. The mean of the MLD-averaged data is the depth-averaged temperature, but the matched data and in situ measurements may be from any depth. For this reason, the MLD-averaged mean model temperature was consistently higher than the in situ and the mean matched temperature. This shift is also visible in the difference in the slope and $y$-intersect, $\hat{\beta}_{1}$ and $\hat{\beta}_{0}$, in Table 6 . As the model surface temperature is forced using reanalysis based on aggregated observational data, it is not surprising that the temperature in Table 1 shows a strong correlation between the model and the in situ data. However, the atmospheric forcing dataset, ERA40-reanalysis (Uppala et al., 2005), is a meteorological surface dataset, whereas the in situ measurements and hence the matched model data may occur at any depth. As such, the success of the model is due to its own merit, instead of the similarities between the forcing and in situ datasets. Both the matched model and the MLD-averaged data capture the 1990 in situ peak and subsequent rise in Fig. 12. This suggests that some of the increase seen in the mean in situ temperature between 1993 and 2005 is not an artefact of uneven

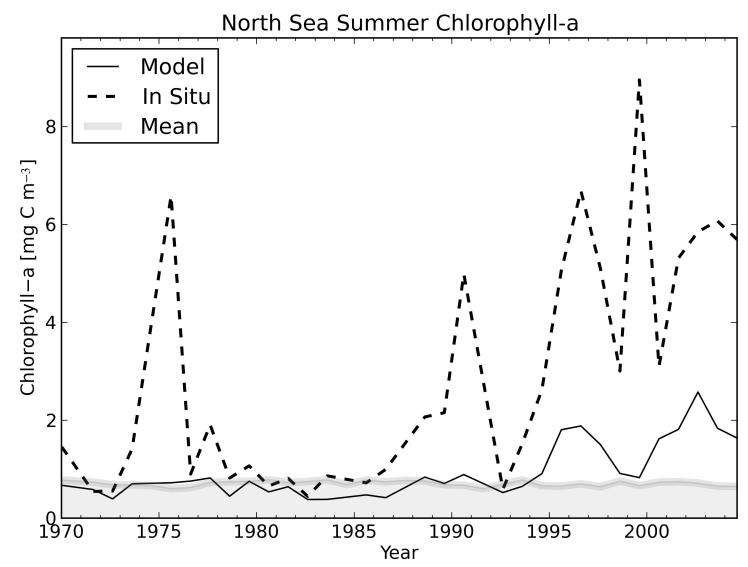

Fig. 17. North Sea mean summer chlorophyll time series: the black line is the matched model mean summer chlorophyll, the dashed line the mean summer in situ chlorophyll, and the grey area shows the MLD-averaged mean chlorophyll of the North Sea.

Table 6. Linear regression output parameters for temperature $(T)$ and salinity (Sal.). The parameters shown are the slope of the line $\left(\hat{\beta}_{1}\right)$, the y-axis intersect $\left(\hat{\beta}_{0}\right)$, the correlation coefficient $(R)$, and the two-tailed probability $(P)$, the standard error $(\epsilon)$, and the number of data $(N)$.

\begin{tabular}{cccc}
\hline & & MLD averaged & Matched \\
\hline & $\hat{\beta}_{1}$ & 0.4495 & 0.9565 \\
Annual & $\hat{\beta}_{0}$ & 6.02 & 0.6754 \\
$T$ & $R$ & 0.643 & 0.9572 \\
& $P$ & $<10^{-4}$ & $<10^{-4}$ \\
& $\epsilon$ & 0.0932 & 0.0469 \\
\hline \multirow{2}{*}{ Annual } & $\hat{\beta}_{1}$ & 0.2063 & 1.268 \\
Sal. & $\hat{\beta}_{0}$ & 27.11 & -9.221 \\
& $R$ & 0.3401 & 0.7558 \\
& $P$ & 0.0456 & $<10^{-4}$ \\
& $\epsilon$ & 0.0993 & 0.1783 \\
\hline
\end{tabular}

sampling, but rather physically observable inter-annual variability.

Figure 13 is a time-series plot of the annual mean North Sea salinity. The linear regression results associated with this plot are shown in the "annual" column of the salinity of Table 2. All the time granularities show a strong correlation $(R>0.75)$ between the matched model and the in situ salinity. The MLD-averaged mean model salinity is consistently lower than the in situ and the matched salinity, but displayed some skill in reproducing the overall trend. The matched model data here indicate that the model reproduced an arbitrary set of in situ measurements with moderate success. This success allows some confidence that the mean state of the model salinity is a fair representation of the mean state of the system salinity. 
Table 7. Linear regression output parameters for nitrates $\left(\mathrm{NO}_{3}\right)$, phosphates $\left(\mathrm{PO}_{4}\right)$ and chlorophyll (Chl.). The parameters shown are the slope of the line $\left(\hat{\beta}_{1}\right)$, the $\mathrm{y}$-axis intersect $\left(\hat{\beta}_{0}\right)$, the correlation coefficient $(R)$, and the two-tailed probability $(P)$, the standard error $(\epsilon)$, and the number of data $(N)$.

\begin{tabular}{clcc}
\hline & & MLD averaged & Matched \\
\hline & $\hat{\beta}_{1}$ & 0.0295 & 1.336 \\
Winter & $\hat{\beta}_{0}$ & 13.64 & -10.05 \\
$\mathrm{NO}_{3}$ & $R$ & 0.3781 & 0.8191 \\
& $P$ & 0.0251 & $<10^{-4}$ \\
& $\epsilon$ & 0.0126 & 0.1518 \\
\hline & $\hat{\beta}_{1}$ & 0.0229 & 0.938 \\
Winter & $\hat{\beta}_{0}$ & 0.7217 & -0.0255 \\
PO $_{4}$ & $R$ & 0.1602 & 0.5799 \\
& $P$ & 0.3654 & 0.0001 \\
& $\epsilon$ & 0.0249 & 0.2167 \\
\hline & $\hat{\beta}_{1}$ & 0.007 & 1.62 \\
Spring & $\hat{\beta}_{0}$ & 1.966 & 0.2675 \\
Chl. & $R$ & 0.1473 & 0.37 \\
& $P$ & 0.4212 & 0.0263 \\
& $\epsilon$ & 0.0085 & 0.6975 \\
\hline \multirow{2}{*}{ Summer } & $\hat{\beta}_{0}$ & 0.7444 & -0.0887 \\
Chl. & $R$ & -0.5414 & 0.7083 \\
& $P$ & 0.002 & $<10^{-4}$ \\
& $\epsilon$ & 0.0033 & 0.5515 \\
\hline & & &
\end{tabular}

The mean winter North Sea nitrates are shown in Fig. 14, and the mean winter North Sea phosphates are shown in Fig. 15. These plots show that the model had significant skill in reproducing the in situ nitrate and phosphate measurements, but only once unpaired model cells were masked. The winter nitrate linear regression fit was consistent with a line of unity slope, and had a correlation coefficient of $R=0.8191$. This correlation was not present in the unmatched mixed layer depth-averaged model nitrates in Table 3 or Fig. 14, suggesting that the bulk of the inter-annual variability of in situ nitrates is a result of sampling. The other time selections and granularities of the nitrates in Table 3 indicate that the inter-annual variability of in situ nitrates was reasonably reproduced under other time granularities and selections. However, the winter nutrient behaviour is arguably more important than the rest of the years as the winter nutrients determine the resources available for the spring phytoplankton bloom. The winter phosphate linear regression fit was consistent with a line of unity slope and a null intersect, but this skill was not present in the spring and summer phosphates in Table 4. The annual summer phosphate column of this table shows an instance of the model and the in situ data match breaking down; the correlation and slope are both very close to zero, and the $p$ value is nearly unity. However, the nutrients are depleted in the summer, and the results

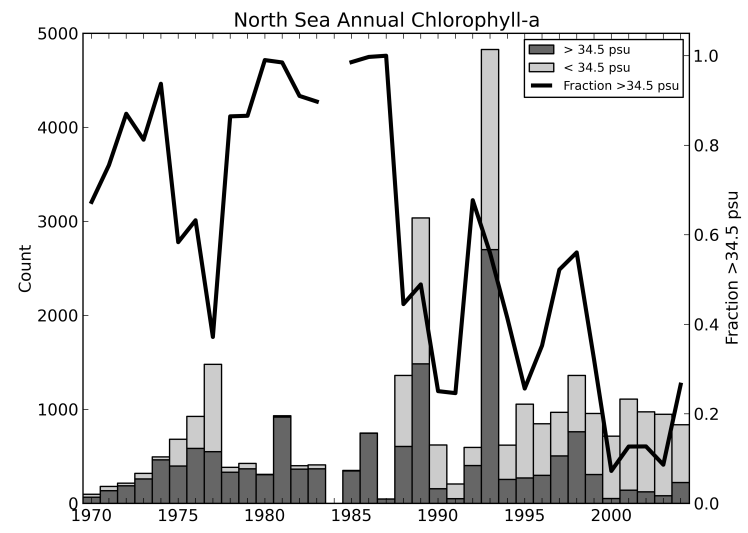

Fig. 18. The number of North Sea in situ chlorophyll measurements, grouped into a high salinity (Sal. $>34.5 \mathrm{psu}$ ) in dark grey and low salinity (Sal. $<34.5 \mathrm{psu}$ ) in light grey. The dark line shows the fraction of high salinity. There is a gap in 1984 due to the absence of simultaneous measurements of chlorophyll and salinity.

of a linear regression would be skewed towards large outlying in situ measurements, which would never be reproduced by the model. Secondly, winter phosphate has a much larger impact on the ecosystems annual cycle, and its successful simulation by the model has more importance.

Figures 14 and 15 both show a large peak in 1983. In the winter of 1983, almost all North Sea nitrate and phosphate measurements in the ICES database were taken in coastal environments. The peaks are also present in the matched model data, but not in the mean of the MLD-averaged nitrates and phosphates. The presence of these peaks in both model and measurement suggests that the bulk of the variability of in situ nitrates and phosphates is due to uneven coverage, rather than inter-annual variability. Due to the incongruities of historic in situ data such as these peaks, model validators should be extremely cautious to ensure that their validation compares like-datasets to each other.

Three time series figures are shown for the North Sea chlorophyll: Fig. 16 shows the mean spring chlorophyll; Fig. 17 shows the mean summer chlorophyll; and Fig. 18 show the total number of chlorophyll measurements per year grouped into high salinity and low salinity categories. The offshore high salinity region cut-off of 34.5 psu was taken from OSPAR Commission (2008). This figure was made by matching up the chlorophyll and salinity ICES datasets, but this process is not $100 \%$ efficient because there are chlorophyll measurements with no corresponding salinity measurement. A large increase in the mean value of the in situ chlorophyll but not in the model chlorophyll can be seen after 1993 in the first two chlorophyll figures. As shown in Fig. 18, these years correspond to years in which much of in situ data were taken in low salinity water. Furthermore, these estuarine and coastal regions have high variability in chlorophyll and salinity that the model is unable to capture due to the relatively low spatial resolution. To summarise, the bulk of the 


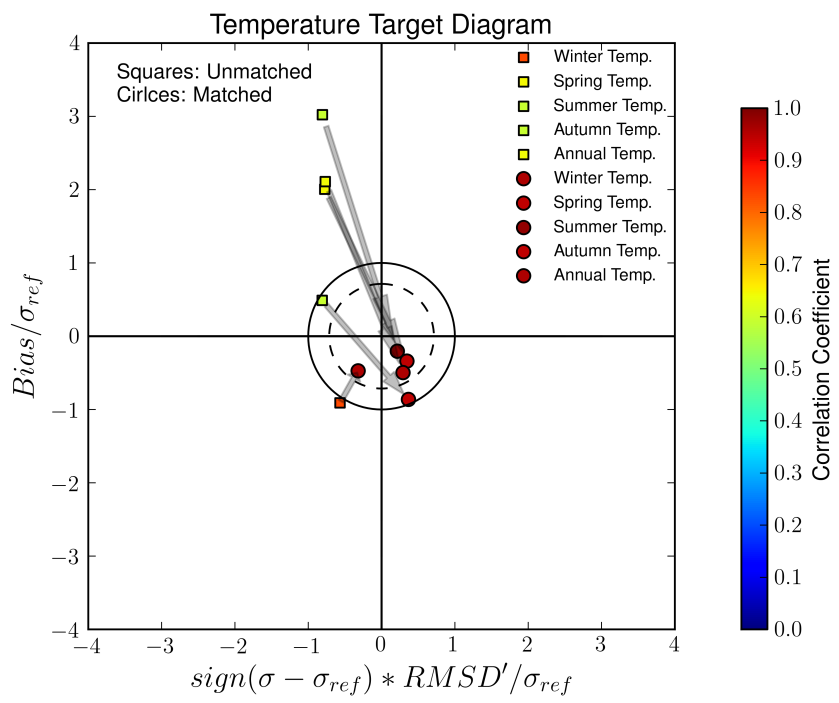

Fig. 19. Target diagram showing the impact of the transition from the unmatched to the matched methods on temperature.

chlorophyll data after 1993 was measured where the model is less effective.

Despite these limitations, the matched model data reproduced the variability of the in situ chlorophyll measurements with a correlation of $R=0.708$ in the annual summer. The matched model data did not produce a significant correlation with the spring in situ data. This can be explained by the greater chlorophyll variability in the spring, and the high sensitivity to bloom timing. A small difference in the model bloom timing relative to nature will result in a large residual. Additionally, as the seasons were defined as strict threemonth periods, it is possible that, for the earliest blooms, for instance in a coastal area, the springtime bloom may have overflowed into the wintertime bin. This bin edge overflow is a more critical issue for chlorophyll linear regression due to its rapid blooms than for temperature, which is much smoother. It is also possible that some of the in situ chlorophyll measurements were biased towards regions that were biologically active.

Although much of the in situ variability of the larger datasets (temperature, salinity, nitrates, and phosphates) can be accounted for by the model, POLCOMS-ERSEM does not reproduce many of the historic trends of the in situ chlorophyll measurements on a point-to-point basis. While a more diverse distribution of North Sea chlorophyll measurements would help to validate the model or produce new biogeochemical parametrisations, it is not possible to travel back in time to obtain such a dataset. The failure to reproduce the historic data time series may be due to the effects of sub-pixel variability, in which case higher resolution models could allow a point-to-point study of chlorophyll to converge on the in situ measurements. It is also possible that a better match between the in situ measurements and the model may yet

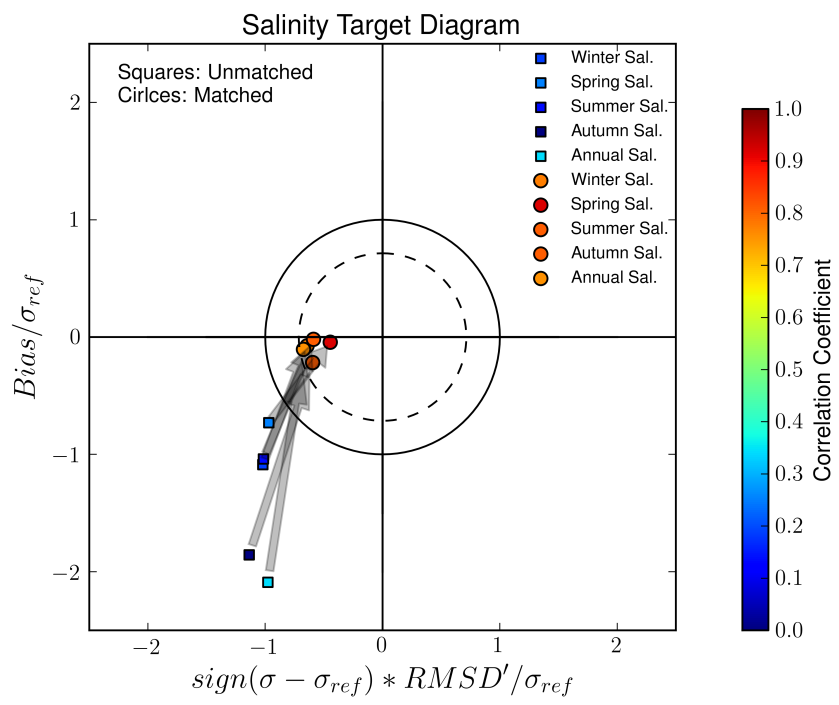

Fig. 20. Target diagram showing the impact of the transition from the unmatched to the matched methods on salinity.

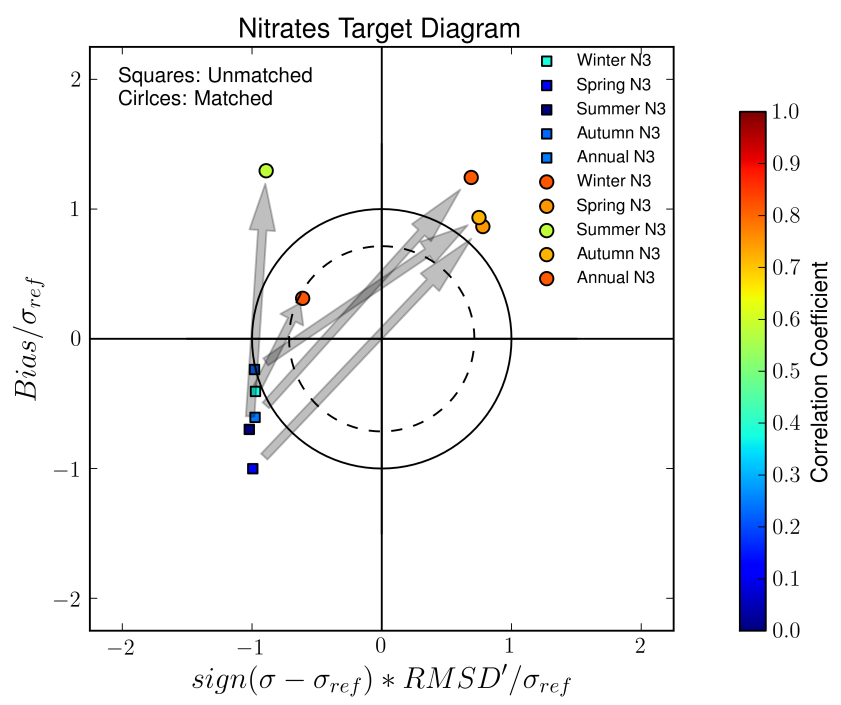

Fig. 21. Target diagram showing the impact of the transition from the unmatched to the matched methods on nitrates.

occur through improvements to the phytoplankton parametrisation.

The number of data is also shown in the row labelled $N$ of Tables 1-5. Of the five datasets used in this study, chlorophyll is the smallest by approximately a factor of four. It is important to note that the number of in situ data is completely independent of the quality of the model. While additional in situ measurements could be used to improve the parametrisation of the model for a future model run, more in situ measurements would not directly result in a higher correlation or a better match using the point-to-point matching. Conversely, this means that the point-to-point matching method does not have any data quantity or distribution requirements. 


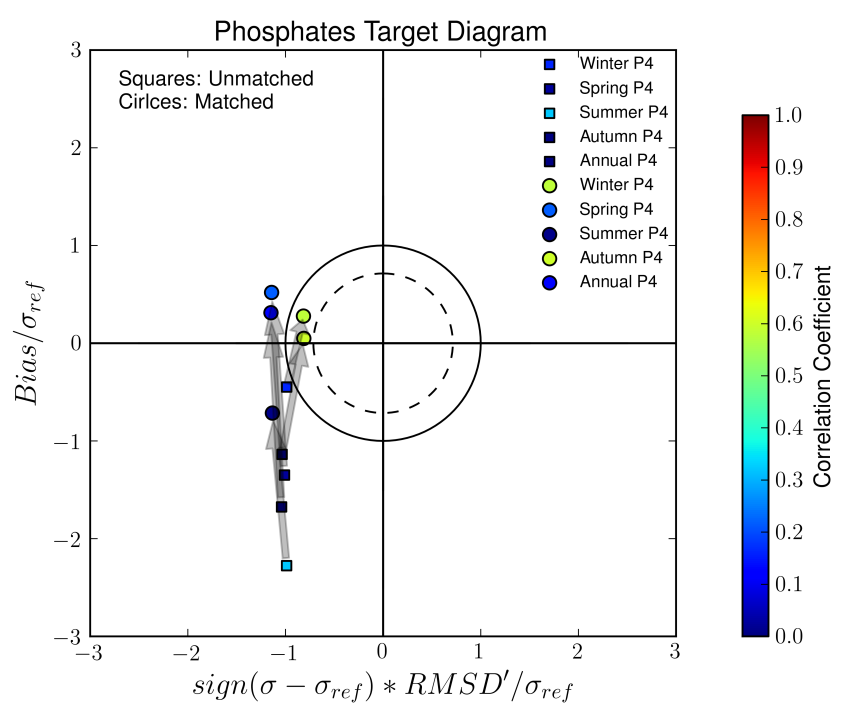

Fig. 22. Target diagram showing the impact of the transition from the unmatched to the matched methods on phosphates.

Figures 19-23 are target diagrams (Jolliff et al., 2009) showing the pattern statistics for each of the five datasets. The $\mathrm{x}$-axis shows the normalised unbiased root mean square difference $\left(\mathrm{RMSD}^{\prime}\right)$, and the $\mathrm{y}$-axis shows the normalised bias. Normalisation is performed by dividing by the reference standard deviation, $\sigma_{\text {ref }}$, which is the standard deviation of the in situ data. The diagram's two large circles correspond to lines of constant root mean square difference (RMSD): the outer has RMSD $=1.0$, and the dashed inner circle has an RMSD $=0.71$. In these plots, the square markers describe the comparison of the MLD-averaged data to the mean of the in situ data (unmatched). The round markers are the pattern statistics of the matched model data against the mean of the in situ data (matched). The grey arrows indicate the change due to moving the unmatched to the matched methods. In all the target diagrams, the annual mean time granularity was used because of the absence of the MLD-averaged data with full time granularity. The colour scale of the markers shows the correlation coefficient. As with most targets, the best outcomes occur closer to the centre of the target.

Figures 19 and 20 are concise plots showing change due to the application of the matching method to temperature and salinity data. Reflecting the conclusions of Figs. 12 and 13 , the matching significantly improved the correlation, and the normalised bias and $\mathrm{RMSD}^{\prime}$, moving all temperature and salinity markers closer to the centre. The salinity target diagram is particularly impressive as it shows a large decrease in the bias, and a large increase in correlation due to going from an unmatched comparison to a point-to-point comparison.

Although winter nitrates in Fig. 21 show the best improvement in bias and RMSD of that figure, all time selections show unambiguous increases in correlation, shifting from cold colours to hot colours. The other time selections also

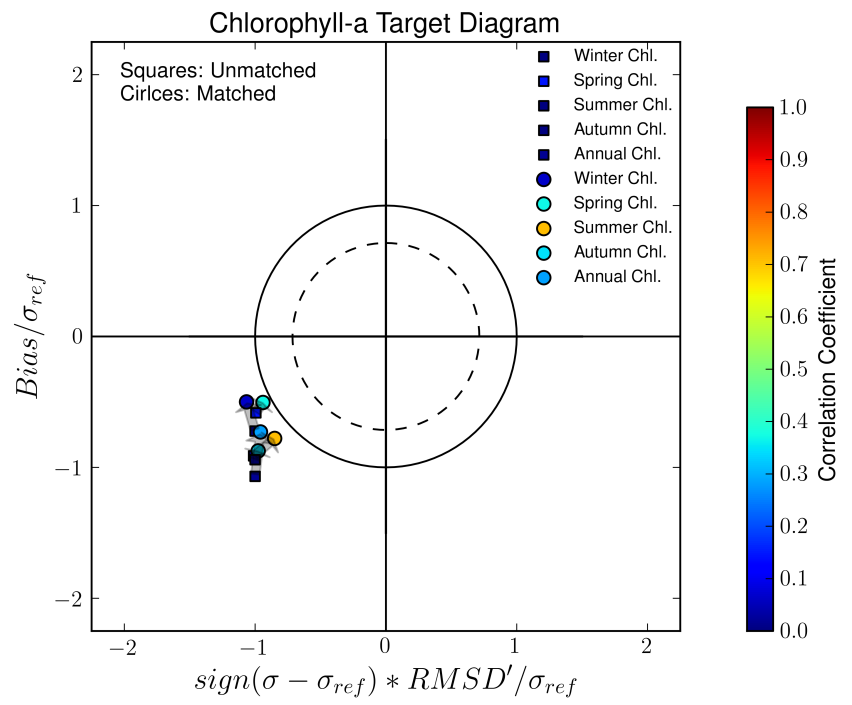

Fig. 23. Target diagram showing the impact of the transition from the unmatched to the matched methods on chlorophyll $a$.

show substantial shifts; the markers move across the diagram while maintaining an approximately constant RMSD.

In Fig. 22, the autumn and winter phosphate time selections both moved inside the RMSD $=1.0$ circle and increased in correlation. The other phosphate time selections maintained similar unbiased RMSD' while decreasing their normalised bias.

In terms of the chlorophyll $a$, Fig. 23 shows that matching does not produce the dramatic shifts seen in the other measurements. However, it is clear from the legend that the match increased the chlorophyll correlation, except for in the winter. The normalised bias decreased in all time selections, and the unbiased RMSD' decreased in all time selections but winter.

These figures illustrate the importance of the matching method in at least two ways. Firstly, a model may seemingly fail to reproduce the mean state of the system, when it is the in situ data that are not representative of the mean state of a system. For instance, in Fig. 20, the unmatched comparison barely reaches a correlation of $R=0.4$, while the matched comparison has high correlation and RMSD $>0.71$. Secondly, the mean state of the model may appear to underestimate or overestimate an in situ dataset, even when the opposite is true. For instance, in Fig. 21, all unmatched points have a negative normalised bias, and the unmatched model appears to underestimate the in situ nitrates. However, the matched comparisons all have a normalised bias greater than zero, and the model appears to overestimate the in situ nitrate. 


\section{Conclusions}

A point-to-point method was presented as a tool to validate a marine biogeochemical model hindcast of the POLCOMSERSEM model with sparse historic CTD and low-resolution bottle in situ measurements. To demonstrate the method, in situ temperature, salinity, nitrates, phosphates, and chlorophyll $a$ from the North Sea were compared against both the point-to-point model data and the annual and seasonal means.

Firstly, the point-to-point method was used to show that POLCOMS-ERSEM displayed skill at reproducing all five of the variables. POLCOMS-ERSEM is most successful at reproducing the physical variables (temperature and salinity) and is least successful at reproducing the biological variable chlorophyll on a pixel by pixel basis. The model had moderate skill at reproducing the nutrients phosphate and nitrate, especially in the winter. This is to be expected, as the physical variables are relatively deterministic, tractable and straightforward to calculate. While the chlorophyll in situ dataset was only reproduced with moderate success, due to the complex and highly variable biological network, even modest success at reproducing chlorophyll trends should be heralded as a victory.

Secondly, using time series plots, it was shown that POLCOMS-ERSEM has significant skill in reproducing the inter-annual variability of some policy-driven time selections of in situ datasets. It also became apparent that the bulk of the variability in the in situ measurements may be due to uneven and low coverage, rather than inter-annual variability of the mean state of the system. For these reasons, we recommend that in situ datasets such as these should be used with caution in trend and inter-annual variability studies.

Thirdly, target diagrams were used to identify some of the strengths and weaknesses of the matching method. It was found that the matching method does not always produce simultaneous improvements to bias, root mean square difference and correlation. However, improvements in bias, $\mathrm{RMSD}^{\prime}$ and correlation relative to the unmatched model were observed in most cases studied here. These improvements are not guaranteed nor are they the principal motivation for the use of the point-to-point method. The point-to-point method is simply a better method to exploit sparse, uneven in situ data while compensating for its variability.

The ICES datasets have been shown to be useful for model validation, and their limitations do not hinder point-to-point matching. Nevertheless, there is a need for larger and longer term non-coastal datasets, which could perhaps be fulfilled in part through the use of next-generation Bio-Argo floats or wave gliders. In addition to validating the model ability, these datasets are required to understand model behaviour and, consequently, to plan next-generation model development and validation. Future in situ datasets should strive for consistent multi-decadal coverage and good representation of both coastal and offshore environments.
Finally, it is important to remember that historic datasets were not recorded for the purpose of model validation; they have limits. As such, it is crucial to account for these restrictions when validating hindcasts. When performing a model validation using a direct comparison, it is necessary to process the model data to resemble the in situ dataset as much as possible. If a direct comparison validation is performed without some kind of matching, the predictive power of the model could be seriously misjudged.

Acknowledgements. This work is supported by the NERC National Capability in Modelling programme at Plymouth Marine Laboratory and Theme 6 of the EC seventh framework program through the Marine Ecosystem Evolution in a Changing Environment (MEECE No. 212085) Collaborative Project.

Edited by: A. Stenke

\section{References}

Arhonditsis, G. B. and Brett, M. T.: Evaluation of the current state of mechanistic aquatic biogeochemical modelling, Mar. Ecol. Prog. Ser., 271, 13-26, doi:10.3354/meps271013, 2004.

Blackford, J. C., Allen, J. I., and Gilbert, F. J.: Ecosystem dynamics at six contrasting sites: a generic modelling study, J. Marine Syst., 52, 191-215, doi:10.1016/j.jmarsys.2004.02.004, 2004.

Doney, S. C., Lima, I., Moore, J. K., Lindsay, K., Behrenfeld, M. J., Westberry, T. K., Mahowald, N., Glover, D. M., and Takahashi, T.: Skill metrics for confronting global upper ocean ecosystem-Biogeochemistry models against field and remote sensing data, J. Marine Syst., 76, 95-112, doi:10.1016/j.jmarsys.2008.05.015, 2009.

Edwards, K. P., Barciela, R., and Butenschön, M.: Validation of the NEMO-ERSEM operational ecosystem model for the North West European Continental Shelf, Ocean Sci., 8, 983-1000, doi:10.5194/os-8-983-2012, 2012.

FAO: FAO Major Fishing Areas, (Major Fishing Area 27), CWP Data Collection, available at: http://www.fao.org/fishery/area/ Area27/en\#NB04F5, (last access: 17 August 2012), 2008.

Garcia, H. E. and Levitus, S.: World Ocean Atlas 2005, vol. 4, Nutrients (phosphate, nitrate, silicate), Tech. Rep. 64, National Oceanographic Data Centre (US), Ocean Climate Laboratory, Washington, DC, 2010a.

Garcia, H. E. and Levitus, S.: World Ocean Atlas 2005, vol. 3, Dissolved oxygen, apparent oxygen utilisation, and oxygen saturation, Tech. Rep. 63, National Oceanographic Data Centre (US), Ocean Climate Laboratory, Washington, DC, $2010 \mathrm{~b}$.

Holt, J., James, I. D., and Jones, J. E.: An s coordinate density evolving model of the northwest European continental shelf 2, Seasonal currents and tides, J. Geophys. Res., 106, 14035-14053, doi:10.1029/2000JC000303, 2001.

Holt, J., Butenschön, M., Wakelin, S. L., Artioli, Y., and Allen, J. I.: Oceanic controls on the primary production of the northwest European continental shelf: model experiments under recent past conditions and a potential future scenario, Biogeosciences, 9, 97-117, doi:10.5194/bg-9-97-2012, 2012. 
ICES: ICES Dataset on Ocean Hydrography, The International Council for the Exploration of the Sea Copenhagen, Copenhagen, 2009.

Jöckel, P., Kerkweg, A., Pozzer, A., Sander, R., Tost, H., Riede, H., Baumgaertner, A., Gromov, S., and Kern, B.: Development cycle 2 of the Modular Earth Submodel System (MESSy2), Geosci. Model Dev., 3, 717-752, doi:10.5194/gmd-3-717-2010, 2010.

Jolliff, J. K., Kindle, J. C., Shulman, I., Penta, B., Friedrichs, M. A., Helber, R., and Arnone, R. A.: Summary diagrams for coupled hydrodynamic-ecosystem model skill assessment, J. Marine Syst., 76, 64-82, doi:10.1016/j.jmarsys.2008.05.014, 2009.

Lewis, K., Allen, J., Richardson, A. J., and Holt, J.: Error quantification of a high resolution coupled hydrodynamicecosystem coastal-ocean model: Part 3, validation with continuous plankton recorder data, J. Marine Syst., 63, 209-224, doi:10.1016/j.jmarsys.2006.08.001, 2006.

OSPAR Commission: Common Procedure for the Identification of the Eutrophication Status of the OSPAR Maritime Area, UK National Report, London, UK, 2008.

Robeson, S.: Influence of spatial sampling and interpolation on estimates of air temperature change, Clim. Res., 4, 119-126, 1994.

Saux Picart, S., Butenschn̈, M., and Shutler, J. D.: Wavelet-based spatial comparison technique for analysing and evaluating twodimensional geophysical model fields, Geosci. Model Dev., 5, 223-230, doi:10.5194/gmd-5-223-2012, 2012.
Stow, C. A., Jolliff, J., Mcgillicuddy, D. J., Doney, S. C., Allen, J. I., Friedrichs, M. A. M., Rose, K. A., and Wallhead, P.: Skill assessment for coupled biological/physical models of marine systems, J. Marine Syst., 76, 4-15, doi:10.1016/j.jmarsys.2008.03.011, 2009.

Taylor, K. E.: Summarizing multiple aspects of model performance in a single diagram, J. Geophys. Res., 106, 7183-7192, 2001.

Uppala, S. M., KÅllberg, P. W., Simmons, A. J., Andrae, U., Bechtold Da Costa, V., Fiorino, M., Gibson, J. K., Haseler, J., Hernandez, A., Kelly, G. A., Li, X., Onogi, K., Saarinen, S., Sokka, N., Allan, R. P., Andersson, E., Arpe, K., Balmaseda, M. A., Beljaars, A. C. M., Berg, L. Van De, Bidlot, J., Bormann, N., Caires, S., Chevallier, F., Dethof, A., Dragosavac, M., Fisher, M., Fuentes, M., Hagemann, S., Hólm, E., Hoskins, B. J., Isaksen, L., Janssen, P. A. E. M., Jenne, R., Mcnally, A. P., Mahfouf, J.-F., Morcrette, J.-J., Rayner, N. A., Saunders, R. W., Simon, P., Sterl, A., Trenberth, K. E., Untch, A., Vasiljevic, D., Viterbo, P., and Woollen, J.: The ERA-40 re-analysis, Q. J. Roy. Meteorol. Soc., 131, 2961-3012, doi:10.1256/qj.04.176, 2005.

Vörösmarty, C. J., Fekete, B. M., Meybeck, M., and Lammers, R. B.: Global system of rivers: its role in organizing continental land mass and defining land-to-ocean linkages, Global Biogeochem. Cy., 14, 599-621, doi:10.1029/1999GB900092, 2000.

Young, E. F. and Holt, J. T.: Prediction and analysis of long-term variability of temperature and salinity in the Irish Sea, J. Geophys. Res., 112, C01008, doi:10.1029/2005JC003386, 2007. 